NBER WORKING PAPER SERIES

\title{
ADVANCE MARKET COMMITMENTS: \\ INSIGHTS FROM THEORY AND EXPERIENCE
}

\author{
Michael Kremer \\ Jonathan D. Levin \\ Christopher M. Snyder \\ Working Paper 26775 \\ http://www.nber.org/papers/w26775 \\ NATIONAL BUREAU OF ECONOMIC RESEARCH \\ 1050 Massachusetts Avenue \\ Cambridge, MA 02138 \\ February 2020
}

The authors were members of the Economic Expert Group that helped to design the pilot Advance Market Commitment for pneumococcus vaccine. We are grateful to Arthur Baker and Junyi Que for excellent research assistance and Owen Barder, Rachel Glennerster, Imran Rasul, and participants in "The Economics of Health Epidemics" session at the 2020 American Economic Association meetings for insightful comments. This working paper integrates the text and online appendix of a paper forthcoming in the American Economic Association Papers and Proceedings. The views expressed herein are those of the authors and do not necessarily reflect the views of the National Bureau of Economic Research.

At least one co-author has disclosed a financial relationship of potential relevance for this research. Further information is available online at http://www.nber.org/papers/w26775.ack

NBER working papers are circulated for discussion and comment purposes. They have not been peer-reviewed or been subject to the review by the NBER Board of Directors that accompanies official NBER publications.

(C) 2020 by Michael Kremer, Jonathan D. Levin, and Christopher M. Snyder. All rights reserved. Short sections of text, not to exceed two paragraphs, may be quoted without explicit permission provided that full credit, including $\odot$ notice, is given to the source. 
Advance Market Commitments: Insights from Theory and Experience

Michael Kremer, Jonathan D. Levin, and Christopher M. Snyder

NBER Working Paper No. 26775

February 2020

JEL No. I15,L65,O38

\section{ABSTRACT}

Ten years ago, donors committed $\$ 1.5$ billion to a pilot Advance Market Commitment (AMC) to help purchase pneumococcal vaccine for low-income countries. The AMC aimed to encourage the development of such vaccines, ensure distribution to children in low-income countries, and pilot the AMC mechanism for possible future use. Three vaccines have been developed and more than 150 million children immunized, saving an estimated 700,000 lives. This paper reviews the economic logic behind AMCs, the experience with the pilot, and key issues for future AMCs.

Michael Kremer

Harvard University

Department of Economics

Littauer Center M20

Cambridge, MA 02138

and NBER

mkremer@fas.harvard.edu

Jonathan D. Levin

Stanford University

Department of Economics

Stanford, CA 94305-6072

and NBER

jdlevin@stanford.edu
Christopher M. Snyder

Department of Economics

Dartmouth College

301 Rockefeller Hall

Hanover, NH 03755

and NBER

chris.snyder@dartmouth.edu

A data appendix is available at http://www.nber.org/data-appendix/w26775 


\section{Introduction}

Ten years ago, donors committed $\$ 1.5$ billion to a pilot Advance Market Commitment (AMC) to help purchase pneumococcal vaccine for low-income countries. The AMC aimed to encourage the development of such vaccines, ensure distribution to children in low-income countries, and pilot The AMC mechanism for possible future use. Three vaccines have been developed and more than 150 million children immunized, saving an estimated 700,000 lives. This paper reviews the economic logic behind AMCs, the experience with the pilot, and key issues for future AMCs.

\section{Review of AMC Theory}

\subsection{Technologically Distant Target}

Kremer and Glennerster (2004) proposed AMCs to encourage research on vaccines against diseases such as malaria primarily affecting low-income countries, and to promote access to these vaccines once developed. The AMC has donors pledge to top up payments for newly introduced vaccine meeting technical benchmarks, conditional on the firm's setting the price close to marginal cost.

The AMC is designed to tackle static and dynamic distortions in the vaccine market. The price-cap feature of the AMC is designed to mitigate static deadweight loss from firms' exercising market power. Donors' commitment to top up price above marginal cost is designed to bolster dynamic R\&D incentives, which are weak due to a number of factors including limited purchasing power in low-income countries, free riding by the unvaccinated on the reduction in disease transmission, and political pressure to reduce price once a vaccine has been developed, causing a hold-up problem.

AMCs' "pull" funding is meant to supplement, not replace, direct R\&D support (i.e., "push" funding) while mitigating problems attendant with trying to pick winning projects ex ante under asymmetric information.

\subsection{Technologically Close Target}

The original AMC concept was translated into an actionable proposal in a Center for Global Development working group report (Barder, Kremer, and Levine 2005). The group suggested 
conducting AMCs for both technologically distant and technologically close targets. There are several important theoretical distinctions between these targets.

First, the further a vaccine is along its development path into clinical trials, the less R\&D remains to be undertaken. The key challenge switches from incentivizing $R \& D$ to incentivizing adequate capacity to bring usage to socially efficient levels. Vaccine capacity is expensive. Whether to avoid hold up of the substantial capacity investment required (in a bargaining model) or to leverage monopsony power (in a price-theoretic model), a robust theoretical finding is that firms will tend to underinvest in capacity absent an AMC.

Second, the nature of the information asymmetry between firms and AMC funders may change with technological distance. For a technologically distant vaccine, the funder may know little about the nature and viability of the technology, giving the researchers asymmetric information about its chance of success. For a technologically close vaccine, published clinical trials may inform the funder about the product's viability, closing that information gap. However, as the firm solidifies its production process, it may instead gain asymmetric information about its costs.

Third, product characteristics are more predictable and easily understood for technologically close products. Country copayments provide an important market test for technologically distant products, preventing resources from being wasted on products meeting technical specifications but not consumer needs. This test may not be needed for technologically close products.

\subsection{Modeling AMC Design}

Kremer, Levin, and Snyder (2019) develop a model of AMC design that illuminates many of these issues. The model focuses on the hold-up problem: firms invest in R\&D and capacity before bargaining with purchasers over price and quantity. Purchasers expropriate some investment returns in bargaining, leading the firm to underinvest. The firm may not develop the vaccine at all; if it does, it will underinvest in capacity to serve low-income countries.

An AMC that commits to a subsidy policy prior to the firm's investment helps address the inefficiency. However, the design matters: if the AMC merely sets a per-dose subsidy, a monopoly supplier can claim the entire AMC fund with its low equilibrium capacity. Increasing capacity 
merely increases the rate at which funds are received. As Kremer, Levin, and Snyder (2019) show, a better solution ties AMC funds to capacity commitments.

\section{Pneumococcal Pilot AMC}

\subsection{Program Launch}

In 2007, five countries and the Gates Foundation pledged \$1.5 billion toward a pilot AMC targeting a pneumococcal conjugate vaccine (PCV). The World Health Organization (WHO) estimated pneumococcus killed more than 700,000 children under five in developing countries annually at that time (WHO 2007). A PCV covering disease strains prevalent in developed countries already existed, and PCVs covering the strains in developing countries were in late-stage clinical trials; so this was a technologically close target.

In 2009, the AMC launched under the supervision of GAVI (formerly the Global Alliance for Vaccines and Immunizations). The design called for firms to compete for ten-year supply contracts capping price at $\$ 3.50$ per dose. A firm committing to supply $X$ million annual doses ( $X / 200$ of the projected 200 million annual need) would secure an $X / 200$ share of the $\$ 1.5$ billion AMC fund, paid out as a per-dose subsidy for initial purchases. The AMC covered the 73 countries below an income threshold for GAVI eligibility. Country co-payments were set according to standard GAVI rules. Countries were required to have $70 \%$ coverage with DPT vaccine to obtain pneumococcus vaccine under the program.

In 2010, GAVI set the first tender for 60 million doses. GSK and Pfizer each committed to supply 30 million doses annually, at $\$ 3.50$ per dose, or $\$ 10.50$ for a three-dose course (these and subsequent facts in this paragraph from GAVI (2019)). GAVI issued subsequent tenders over time, sometimes outpaced by country demand. In each case, the firms expanded their supply commitments in line with the tenders. There has been purchaser and public pressure for the manufacturers to reduce their prices, which currently are down to $\$ 2.90$ per dose. In 2019, a third vaccine developed by the Serum Institute of India qualified for the AMC program. Serum Institute is expected to participate in tenders for the remaining \$262 million of uncommitted AMC funds, reportedly pricing its vaccine at \$2 per dose for low-income countries. 


\subsection{Program Outcomes}

By 2016, PCV was distributed in 60 of the 73 eligible countries, and annual distribution exceeded 160 million doses, enough to immunize over 50 million children per year. As Figure 1 shows, by 2018, nearly half of the target child population in GAVI countries was covered. While coverage is far from full, it is higher than in non-GAVI countries. A key reason why the fraction of the population covered is smaller than the fraction of countries covered is that India, by far the most populous GAVI country, did not adopt PCV until 2017 and currently runs only a limited program in five states.

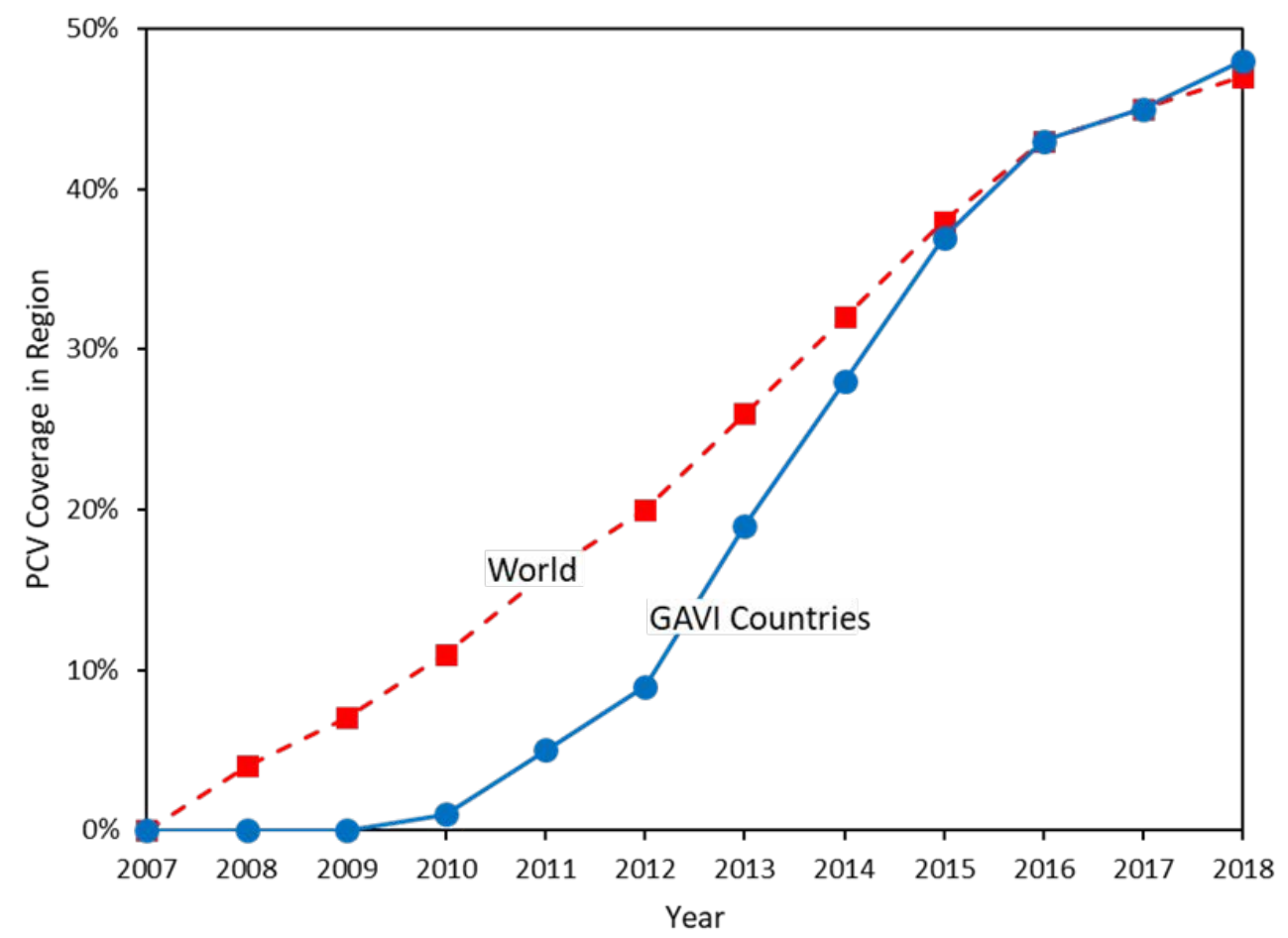

FIGURE 1. PCV COVERAGE IN GAVI COUNTRIES RELATIVE TO WORLD

Notes: Plots of vaccine coverage in 73 GAVI-eligible countries (solid blue line) and in the world (dashed red line). Coverage defined as percentage of children receiving third and final scheduled dose by the nationally recommended age. The figure demonstrates that pneumococcal conjugate vaccine (PCV) coverage in GAVI countries reached nearly $50 \%$ by 2018, surpassing the coverage rate in the rest of the world.

Source: Author calculations using WHO/UNICEF Estimates of National Immunization Coverage (WUENIC) provided on the "Aggregate estimates" worksheet of the coverage_estimates_series.xlsx file downloaded December 18, 2019 from http://www.who.int/immunization/monitoring_surveillance/data/en/.

Estimates from Tasslimi, et al. (2011) suggest that the PCV rollout has been highly cost effective. At initial program prices, the PCV rollout avoided the loss of a disability adjusted life year (DALY) at cost of only $\$ 83$. The WHO classifies interventions as highly cost effective if a 
DALY costs less than one GDP per capita.

Evidence on the cost effectiveness of PCV does not prove the cost effectiveness of the overall AMC because we lack a valid counterfactual. We do not know, absent an AMC, whether and when vaccines would have been developed, how much push funding would have been spent, or what prices would have been set. However, the high cost effectiveness of PCV implies that the AMC would have been worthwhile were there even a small chance that it sped up PCV adoption.

Some insight on the effect of the AMC promoted capacity and adoption can be gained by comparing PCV to rotavirus vaccine, for which GAVI supported purchases over a similar time period without an AMC. ${ }^{1}$ Figure 2 shows that the rate of vaccine coverage in GAVI countries converged to the global rate almost five years faster for PCV. At the same time, shortages of rotavirus vaccine were more severe than of PCV, suggesting firms expanded capacity faster for PCV than rotavirus vaccine.

Appendix A provides details behind calculations showing that if PCV coverage in GAVI countries converged to the global rate at the slower rate of the rotavirus vaccine in Figure 2, 67 million fewer children under age 1 would have been immunized, amounting to a loss of over 12 million DALYs.

\footnotetext{
${ }^{1}$ We selected rotavirus from the six global vaccine initiatives proceeding around that time for the following reasons. Three of them (IPV, second dose of measles, birth dose of hepatitis) involved early-vintage rather than new vaccines. The yellow-fever vaccine was not rolled out in many high-income countries, leaving no good base rate for coverage speed comparison. We conjecture the results would be stronger using HPV, the remaining candidate apart from rotavirus, for comparison, but any slow rollout of HPV vaccine in GAVI countries could be attributed to its administration to older children, slowing coverage expansion.
} 


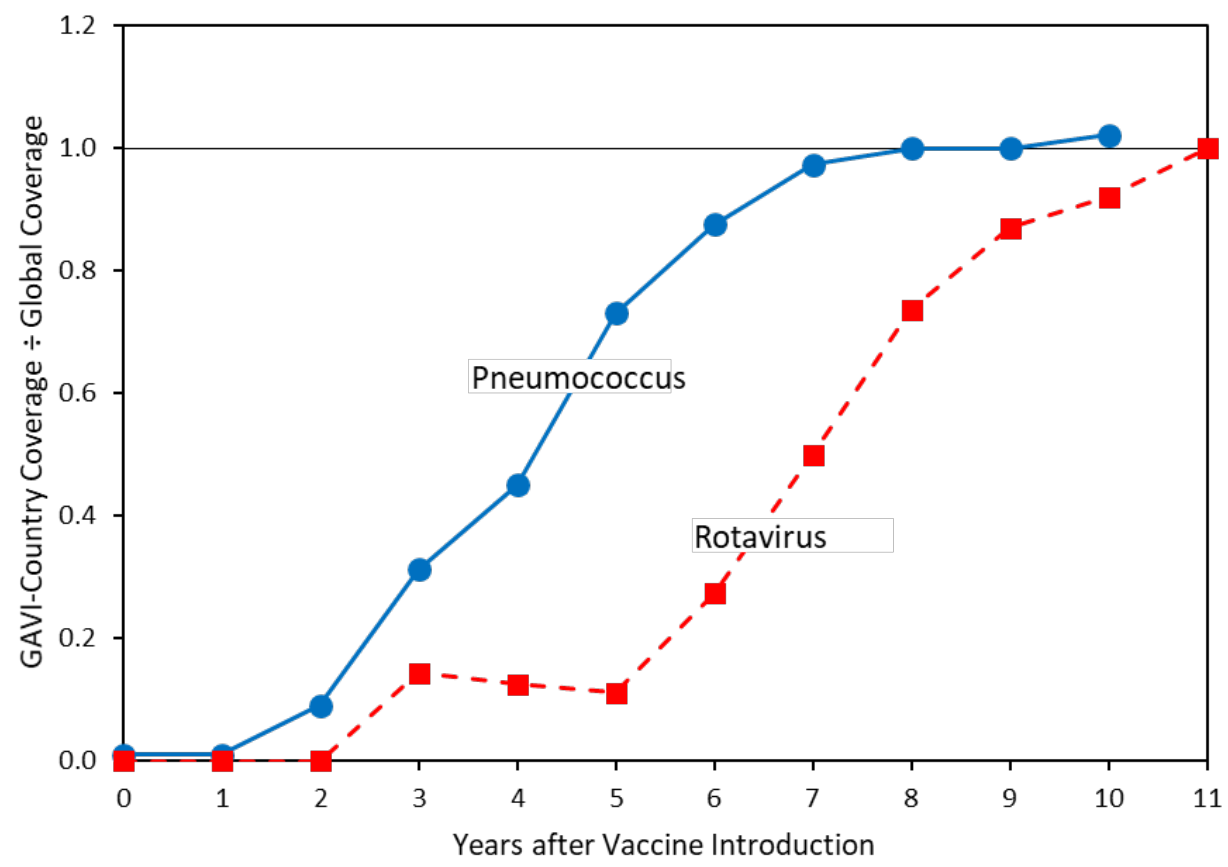

FIGURE 2. COVERAGE FOR VACCINES ROLLED OUT WITH AND WITHOUT AN AMC

Notes: Plots of vaccine coverage in 73 GAVI-eligible countries divided by global coverage. Coverage defined as percentage of children receiving final scheduled dose (three for pneumococcus, two or three for rotavirus depending on schedule) by the nationally recommended age. Each series begins the first year the relevant vaccine was introduced globally: 2008 for pneumococcus and 2006 for rotavirus. Series cut off in 2018 for pneumococcus (year 10 relative to introduction) and 2017 (year 11) for rotavirus. Vaccine coverage in GAVI relative to global coverage represented by the solid blue line for pneumococcal conjugate vaccine (PCV) and by the dashed red line for rotavirus vaccine.

Source: See Figure 1.

\section{Design Issues}

\subsection{Country Copayments, Pricing, Coverage}

AMC designers do not know manufacturers' reservation price for installing adequate capacity to supply needed vaccines or countries' willingness to provide copayments. Because the benefits of vaccines far exceed their production costs, AMC designers face an asymmetric loss function in setting firm prices and country copayments. Offering firms less than their reservation value or asking countries to make copayments greater than their willingness to pay risks children not receiving lifesaving vaccines. This is very costly relative to what the donor can save by paying firms somewhat less or charging countries somewhat more, so maximizing social welfare under uncertainty requires paying firms more than the expected cost of the vaccine and setting country copayments below the expected marginal cost. 
Appendix B provides a range of calibrations quantifying the asymmetry of the loss function. For example, assuming the AMC designer sought to maximize health benefit with the available funds, correctly anticipated the speed of rollout under the AMC, and used money saved from lower vaccine prices for other health interventions at the WHO threshold for highly cost effective interventions, the $\$ 3.50$ price set in the pilot dominates a $\$ 2$ price even if the lower price generated only a $4 \%$ risk the firms refuse to participate (although the precise figure is sensitive to assumptions about alternative uses of savings). Analogous calculations can show that if there were even a small chance that lower copayments would have led India or other countries to adopt sooner, this would have substantially increased the expected number of lives saved through the program.

While some activists have argued that the $\$ 3.50$ paid per dose exceeds manufacturing costs, the relevant issue for AMC designers is not manufacturing costs but firms' reservation values. Their reservation values may substantially exceed manufacturing costs for several reasons: the AMC top up may not fully defray their capacity costs; they may fear that offering a low AMC price would lead higher income countries to press for price reductions.

While these factors imply ex ante optimal prices will exceed expected production costs, the facts that both firms participated even though one likely had substantially higher manufacturing cost and that both continued to participate at $\$ 2.90$ per dose suggests that at least one firm would likely have participated at a lower price.

Still, prices for PCV are much lower under the AMC than outside it. Currently, lowerincome countries in the Americas pay $\$ 12$ or more per dose (WHO 2019); the U.S. Centers for Disease Control and Prevention (CDC) pays \$137 (CDC 2019). As Figure 3 shows, the percentage discount GAVI receives compared to various global price measures is greater for PCV than for almost any other vaccine. 

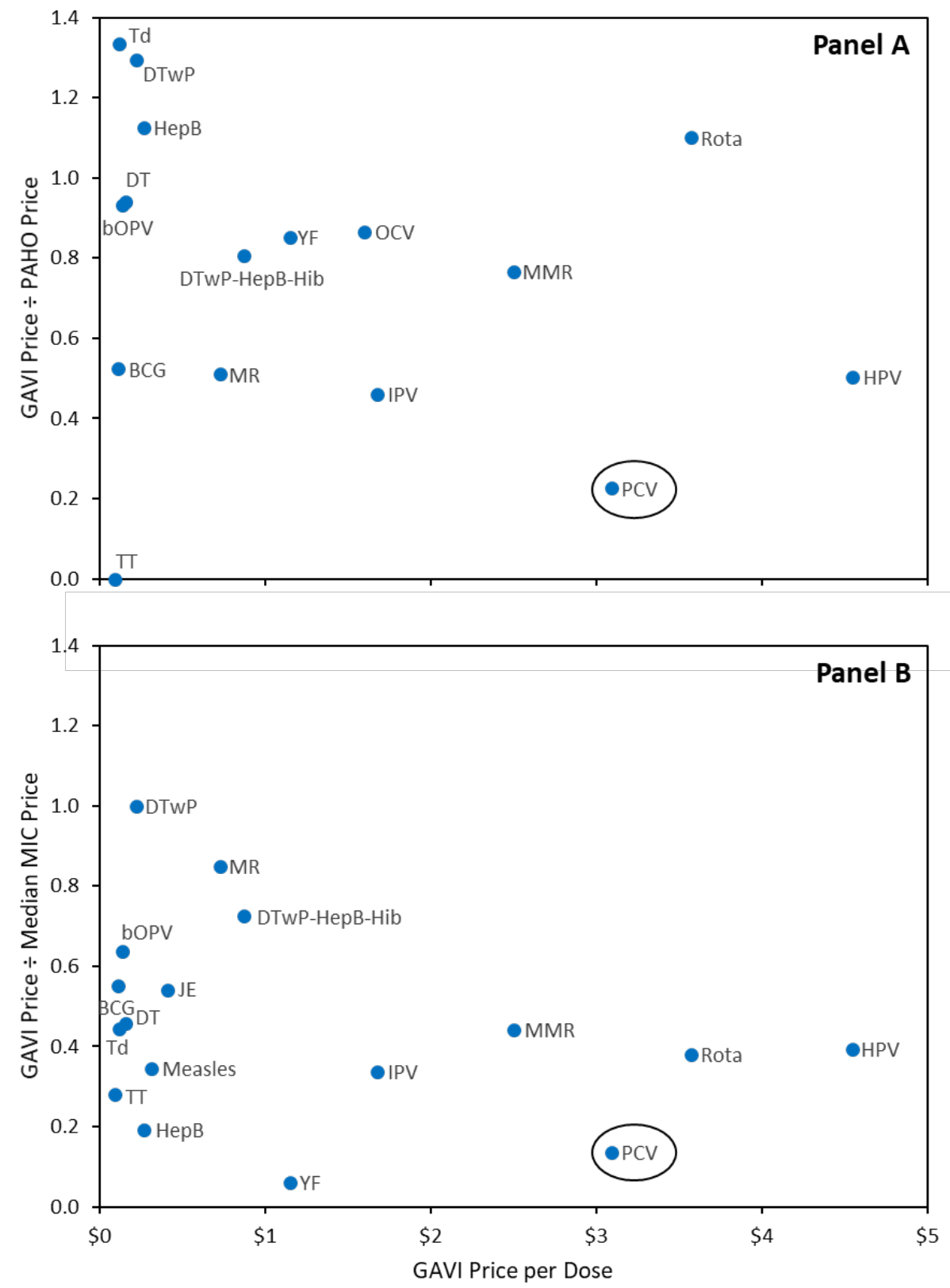

FIGURE 3. GAVI RELATIVE TO WORLD PRICES FOR VACCINES

Notes: Per-dose price paid by GAVI in 2018 plotted against the ratio of the GAVI price to Pan American Health Organization (PAHO) price (Panel A) or the median price paid by a self-procuring middle-income country (Panel B). The pneumococcal conjugate vaccine (PCV) is circled for emphasis.

Source: Author calculations using data from Figure 4.3 of World Health Organization, 2019, "Global Vaccine Market Report,” downloaded January 9, 2020 from http://www.who.int/immunization/programmes_systems/ procurement/mi4a/platform/module2/2019_GlobalVaccine_Market Report.pdf?ua=1. 
Extending AMC participation beyond GAVI-eligible countries could have saved more lives, although it is possible that manufacturers would have demanded higher prices for a program covering higher-income countries if they thought this would have cut into sales at higher prices or put pressure on pricing in higher income countries.

\subsection{Number of Firms}

A program with lower copayments, more generous country inclusion rules, and faster issuance of tenders for the full AMC amount could potentially have generated greater health benefits up to now.

However, holding back some funds for later tenders may have helped incentivize entry by Serum Institute (although the product also received substantial push funding). Serum Institute's vaccine is somewhat cheaper to produce than previous PCVs, but the chief benefit of this entry likely comes from inducing greater competition in the market outside of GAVI countries and in the GAVI-country market after the AMC ends. Another benefit is that India may decide to expand its heretofore limited participation in response to the entry of a domestic supplier.

A key issue for future AMCs will be whether to split the AMC among multiple suppliers and reserve tenders for future entrants as did the pilot pneumococcus AMC or to concentrate incentives on a single supplier as envisioned in Kremer and Glennerster (2004). Sponsors of the AMC pilot prioritized entry of multiple vaccines because they saw competition as essential for holding down long-run prices and avoiding supply interruptions. Kremer and Glennerster (2004) prioritized the development of a vaccine where none currently existed, relying on the price cap, to which the firm agrees to access AMC funds, to keep prices near marginal cost over the long term. Penalty clauses could be specified to mitigate supply interruptions. Development of yet more advanced vaccines could be incentivized through subsequent AMCs.

For distant technological targets, incentivizing a sequence of entrants reduces incentives for the first vaccine to enter. Thus, structuring a program to incentivize multiple entrants may substantially increase total costs. On the other hand, the design and enforcement of long-term contracts that hold prices close to marginal cost and that assure consistent supply through penalty clauses for supply interruptions may be difficult. 


\subsection{Political Economy of Target Choice}

Policymakers may wish to explore future AMCs with different design features. Kremer, Levin, and Snyder (2019) argue that focusing on a technologically more distant target might generate larger overall benefits. However, political factors may favor technologically closer targets. Firms that have a product close to market have stronger incentives to engage politically than firms with early-stage ideas for distant targets. Moreover, while an AMC for a distant product does not impose substantial financial costs unless a product is developed, politicians looking for a "win" may be reluctant to expend significant effort on a project that only pays off once they have left office, if at all.

One solution might be to simultaneously launch a bundle of AMCs that include some eyecatching long-term targets with some short-term targets that will deliver quick wins.

When technologically close targets are chosen, there are further political considerations. There may be strong industry lobbying around product specifications to include their approaches and exclude potential rivals'. If the process involves first selecting a disease target and then designing the AMC details, more weight in the second stage will be put on getting the product to market than saving money that is already earmarked. It may be possible to address some of these concerns by broadening the set of possible targets or having several groups design AMCs targeting different products and using competitive mechanisms to decide which receives funding.

\section{Conclusion}

The AMC moved from theory to practice in its first decade, and we now have a decade of learning from the pneumococcal pilot. While aspects of program evaluation are complicated, the best estimates suggest that the introduction of PCV saved 700,000 lives at a highly favorable cost. Iterations likely could improve AMC design, just as market designs have been refined in settings such as school choice and radio spectrum allocation. Policymakers may wish to consider offering a set of AMCs, perhaps each smaller in scale than the pilot pneumococcus AMC, where potential targets could range beyond health to address agricultural or sustainability problems specific to developing countries. 


\section{References}

Barder, Owen, Michael Kremer, and Ruth Levine. 2005. Making Markets for Vaccines: Ideas to Action. Washington, DC: Center for Global Development.

Centers for Disease Control and Prevention. 2019. CDC Vaccine Price List. https://www.cdc.gov/vaccines/programs/vfc/awardees/vaccine-management/pricelist/index.html.

GAVI. 2019. Advance Market Commitment for Pneumococcal Vaccines: 2018 Annual Report. Geneva.

Kremer, Michael, and Rachel Glennerster. 2004. Strong Medicine: Creating Incentives for Pharmaceutical Research on Neglected Diseases. Princeton: Princeton University Press.

Kremer, Michael, Jonathan Levin, and Christopher M. Snyder. 2019. "Designing an Advanced Market Commitment for New Vaccines,” Harvard University working paper.

Tasslimi Azadeh, et al. 2011. "Cost Effectiveness of Child Pneumococcal Conjugate Vaccination in GAVI-Eligible Countries,” International Health 3: 259-69.

World Health Organization. 2007. "Pneumococcal Conjugate Vaccine for Childhood Immunization-WHO Position Paper,” Weekly Epidemiological Record 82: 93-104. 


\section{Appendix A: Details on PCV-Rotavirus Comparison}

Using the following formula, one can back out the shortfall in immunizations if PCV coverage in GAVI countries converged to the global coverage rate at the slower rate of the rotavirus vaccine:

$$
\sum_{t=2}^{10}\left(\frac{\operatorname{COV}_{p g t}}{\operatorname{COV}_{p w t}}-\frac{\operatorname{COV}_{r g t}}{\operatorname{COV}_{r w t}}\right) \operatorname{COV}_{p w t} U P O P_{g t},
$$

where $\operatorname{COV}_{i j t}$ denotes coverage of the vaccine against disease $i \in\{p, r\}$ and $U P O P_{j t}$ denotes the population under age 1 in location $j \in\{g, w\}$ at each time $t \in\{2,3, \ldots, 10\}$, for $p$ representing pneumococcus, $r$ rotavirus, $g$ GAVI countries, and $w$ the world. For each time $t$ along the horizontal axis of the graph, the factor in parentheses, which is the vertical distance between the graphs, is scaled by PCV coverage in the world and further scaled by the population under age 1 in GAVI countries. The figures for each year are summed to obtain total immunizations.

To convert the immunization shortfall into DALYs lost, the result from equation (1) is multiplied by 3 (PCV doses per immunization) times an estimate of DALYs per PCV dose from the following table based on results from Tasslimi, et al. (2011).

TABLE 1—CALIBRATION OF AMC HEALTH BENEFIT FROM TASSLIMI, ET AL. (2011)

\begin{tabular}{lccc}
\hline \hline Vaccine & DALYs (thousands) & Doses (billions) & DALYs/dose \\
\hline PCV 10 & 106,878 & 1.8 & 0.059 \\
PCV 13 & 119,636 & 1.8 & 0.067 \\
\hline
\end{tabular}

Notes: The 1.8 billion is not the actual number of PCV procured under the AMC program but rather a counterfactual figure used by Tasslimi, et al. (2011) in their cost-effectiveness analysis. They calculate that this is the number of doses required for PCV to have the same rate of infant coverage as DTP-3.

Sources: The first two columns of figures from Table 5 of Tasslimi, et al. (2011). The last column is the quotient of the previous two.

Since the AMC involved about equal distribution of PCV 10 and PCV 13, taking the average of the figures in the last column, one obtains an estimate of 0.063 DALYs saved per PCV dose.

Following these steps, we calculate that if PCV coverage in GAVI countries converged to the global rate at the slower rate of the rotavirus vaccine, 67 million fewer children under age 1 would have been immunized, amounting to a loss of over 12 million DALYs. 


\section{Appendix B: Examples Illustrating Designer's Asymmetric Loss Function}

\section{B1. Introduction}

This appendix provides the details behind the numerical example in the paper illustrating the asymmetry of the Advance Market Commitment (AMC) designer's loss function when trying to set

a) prices offered to manufacturers and

b) copayments required from countries

under uncertainty about these agents' reservation values. The designer's loss from setting too high a manufacturer price is that this increases program expense, diverting resources that could have been used to provide other health benefits. Setting too low a price risks not meeting the firms' reservation values, leading them not to supply pneumococcal conjugate vaccine (PCV) to target countries. As we show, the substantial health benefit provided by PCV relative to alternative uses for the funds - even assuming these funds are used for health interventions that meet the WHO threshold for high cost effectiveness - will lead the loss from not meeting firms reservation values to be asymmetrically large.

Setting the country copayment involves an analogous tradeoff. Setting too low a copayment increases program expense, draining resources from alternative health programs. Setting too high a copayment risks the countries not participating in PCV rollout. Again, the substantial health benefit provided by PCV will lead the latter loss to be asymmetrically large.

To quantify these insights, we will analyze the conditions under which the pilot AMC design would have been improved if the manufacturer price were set below $\$ 3.50$ or country copayment above $\$ 0.20$.

\section{B2. Model}

Suppose the AMC designer sets a manufacturer price $p$ per dose and a copayment $k$ per dose to maximize expected health benefits, recognizing that funds spent on the AMC have an opportunity cost because they can be used in alternative ways.

Let $x(p)$ be the probability that firms are induced to supply PCV under the AMC program, referred to as the participation probability for short. The participation probability is an increasing function of $p$ because higher $p$ is more likely to exceed firms' reservation values, leading them to 
participate in the program. For this exercise, we focus on the effect of $p$ on firms' extensive-margin decision of whether or not to supply the vaccine, implicitly assuming that decisions at the intensive margin such as the scale of capacity expansion or its timing do not depend on $p$. Allowing $p$ to incentivize these intensive-margin decisions in a more general analysis would only strengthen the conclusion about the asymmetry of the loss function. See Kremer, Levin, and Snyder (2019) for an elaborated model capturing incentives on the intensive margin. Denote the complementary probability by $f(p)=1-x(p)$, interpreted as the failure probability, i.e., the probability that the AMC fails to induce firm participation.

Let $q(k)$ denote the number of doses administered under the AMC. This is a decreasing function of the copayment $k$ because higher copayments are more likely to exceed countries' reservation values.

Let $h_{a}$ be the health benefit per dose if firms participate in the AMC, measured in disability-adjusted life years (DALY) saved per dose. Let $h_{o}$ denote the opportunity cost of each dollar spent on the AMC. This opportunity cost is the foregone health benefit from the alternative intervention, measured in DALYs per dollar.

Let $B$ be the total budget available to the AMC designer. The all-in cost of the AMC to the designer per dose (denoted $c$ ), equals the manufacturer price per dose $(p)$, plus the AMC subsidy (denoted $s$ ), plus the administrative costs of distributing the vaccine (denoted $a$ ), minus the country co-payment $(k)$ :

$$
c=p+s+a-k
$$

The AMC designer's objective function (denoted $W$ for welfare) is the expected health benefit that can be generated by its budget:

$$
W=f(p) h_{o} B+x(p)\left\{q(k) h_{a}+[B-q(k) c] h_{o}\right\} .
$$

To derive equation (3), note that with probability $f(p)$, firms decline to provide vaccine at the offered price $p$, in which case the AMC budget $B$ is spent on an alternative intervention which brings health benefit per dollar $h_{o}$ for certain. With probability $x(p)$, firms participate in the AMC. In that case, the AMC designer orders the number of doses, $q(k)$, that adopting countries order. The remaining budget $B-q(k) c$ is spent on the alternative intervention. Equation (3) embodies the assumption that the AMC designer does not value firm profits nor money saved by developing countries in lieu of making copayments. If the designer were to place some welfare weight on 
savings to developing countries from lower copayments, then subsequent calculations would favor lower copayments.

Substituting from equation (2) into (3) and rearranging yields

$$
W=h_{o} B+x(p) q(k)\left[h_{a}-(s+p+a-k) h_{o}\right]
$$

The designer chooses $p$ and $k$ to maximize equation (4).

\section{B3. Parameter Calibration}

The model parameters are calibrated as follows. We fix the AMC subsidy at $s=\$ 0.75$, its average level in the pilot AMC. We fix the administrative cost at a reasonable, round figure of $a=\$ 1.00$. We start in the next section by fixing the country copayment at $k=\$ 0.20$ as in the pilot AMC; the section after that examines the welfare effects of varying $k$. Reflecting the observation that firms did participate at the price offered in the pilot AMC in practice, we assume firms certainly participate at the high AMC price, i.e., we set $x_{0}=1$.

The remaining parameters to calibrate are the health benefits. For $h_{o}$, the health benefit of alternative interventions, we posit a range of values in the sensitivity analysis below. We calibrate $h_{a}=0.063$ as described in the discussion surrounding Table 1 in the previous appendix.

\section{B4. Setting Manufacturer Price}

In this section, we analyze the conditions under which the designer would prefer moving from the price $p_{0}=\$ 3.50$ set in the pilot AMC to a lower per-dose price of $p_{1}=\$ 2.00$. In particular, we solve for the threshold participation probability above which the designer would prefer $p_{1}$ to $p_{0}$, performing sensitivity analysis around various values of the health benefit $h_{o}$ of the alternative intervention

The designer prefers $p_{1}=\$ 2.00$ to $p_{0}=\$ 3.50$ if $W_{1} \geq W_{0}$, where $W_{i}$ here denotes the welfare expression (4) evaluated at price $p_{i}$. Substituting from equation (4) into both sides of the preceding inequality, substituting the indicated prices, and rearranging yields

$$
\frac{x_{0}-x_{1}}{x_{0}} \leq \frac{\left(p_{0}-p_{1}\right) h_{o}}{h_{a}-\left(p_{1}+s+a-k\right) h_{o}}
$$


where $x_{i} \equiv x\left(p_{i}\right)$. The left-hand side equals the proportional reduction in certainty that firms participate in the AMC at the lower price. The numerator on the right-hand side equals the health benefit generated by investing the savings on a PCV dose in the alternative intervention. The denominator equals the health benefit from a PCV dose over the alternative use of this money spent on this dose at the low price, $p_{1}=\$ 2.00$.

The assumption that participation is certain under the original AMC price, i.e., $x_{0}=1$, allows expression (5) to be simplified. Substituting $x_{0}=1$ along with the other calibrated parameters and price values into (5) yields

$$
f_{1} \leq \frac{1.50 h_{o}}{0.063-3.55 h_{o}}
$$

where $f_{i} \equiv 1-x_{i}$. Intuitively, equation (6) says that the designer prefers the lower price only if it does not drive the probability the AMC fails to induce firm participation too high. The fact that the right-hand side of (6) is decreasing in $h_{o}$ implies that an increase in the opportunity cost of funds relaxes the condition, increasing the appeal of a low price. In other words, a reduction in the opportunity cost of funds tightens the condition under which the designer prefers the lower price; lowering the price to $p_{1}=\$ 2.00$ would only make sense if the designer remains fairly certain that firms participate at this lower price. Denote the threshold value of the failure rate at which condition (6) just holds with equality by $\hat{f}_{1}$

Table 2 computes the threshold $\hat{f}_{1}$ for a range of values of $h_{o}$. The first row assumes the cost of saving a DALY through alternative means is $\$ 4,914$, three times per-capita GDP in GAVI countries measured in 2009, the year the AMC was launched. Saving a DALY at three times percapita GDP is the WHO threshold for a cost-effective intervention in a country. This high figure of $\$ 4,914$ translates into a low opportunity cost of AMC funds in the third column, $h_{o}=2.305 \mathrm{e}-4$. With such a low opportunity cost, an AMC price of $p_{1}=\$ 2.00$ is preferred only if there is a less than $\hat{f}_{1}=0.5 \%$ chance (one in two hundred) that firms fail to participate at this lower price. 


\begin{tabular}{llcc}
$\begin{array}{l}\text { Cost effectiveness of } \\
\text { alternative intervention, } \\
1 / h_{o} \text { (\$ per DALY) }\end{array}$ & $\begin{array}{l}\text { Rationale for cost- } \\
\text { effectiveness measure }\end{array}$ & $h_{o}$ & $\begin{array}{c}\text { Threshold failure } \\
\text { rate } \hat{f}_{1}\end{array}$ \\
\hline 4,914 & Three times per-capita GDP & $2.035 \mathrm{e}-4$ & $0.5 \%$ \\
1,638 & Per-capita GDP & $6.105 \mathrm{e}-4$ & $1.5 \%$ \\
1,000 & Higher round figure & $1.000 \mathrm{e}-3$ & $2.5 \%$ \\
500 & Medium round figure & $2.000 \mathrm{e}-3$ & $5.4 \%$ \\
150 & Lower round figure & $6.667 \mathrm{e}-3$ & $25.4 \%$ \\
\hline
\end{tabular}

Notes: The first column contains a range of cost-effectiveness measures for alternative interventions. The second column provides rationales for entries in the first column. The third column is the reciprocal of the first. The last column substitutes the third column into expression (6). In the first row, three times per-capita GDP is the WHO threshold for a cost-effective intervention. In the second row, one times per-capita GDP is the WHO standard for a highly cost-effective intervention. Per-capita GDP is that of GAVI-eligible countries and is measured in 2009, the year of AMC launch.

Sources: GDP figures taken from World Bank, International Comparison Program database.

The second row assumes the cost of saving a DALY thorough alternative means is $\$ 1,638$, equal to per-capita GDP in GAVI countries, the WHO standard for a highly cost-effective intervention. With this opportunity cost, the designer would prefer the $p_{1}=\$ 2.00$ price only if the failure rate were no greater than $\hat{f}_{1}=1.5 \%$.

As the alternative becomes more and more cost effective, the designer requires less and less certain participation to prefer the lower price. The $\$ 1,000, \$ 500$, and $\$ 150$ are round figures for cost effectiveness that trace out a range of possibilities.

\section{B5. Setting Country Copayments}

\section{B5.1. Introduction}

A similar approach can be used to study the country copayment, which the pilot AMC set at \$0.20 per dose. A higher copayment lowers the cost to GAVI and AMC sponsors, but may deter countries from adopting the vaccine. A lower copayment increases the cost but reduces the resources for alternative interventions. The high health benefit of vaccine usage creates an asymmetry between the losses on the two sides that generally favors low copayments.

\section{B5.2. Calibration}

We maintain all the parameter calibrations from before except now we fix the price at the original 
AMC level, $p=\$ 3.50$, and consider reductions in the country copayment from the original level of $k_{0}=\$ 0.20$ to some lower copayment $k_{1}$. Recall that we calibrated the continuation probability as $x(p)=1$, i.e., certain firm participation, when evaluated at the pilot AMC price of $p=\$ 3.50$.

The designer prefers lower copayment $k_{1}$ to $k_{0}=\$ 0.20$ if $W_{1} \geq W_{0}$, where we have redefined $W_{i}$ in this section to denote welfare in equation (4) evaluated at copayment $k_{i}$. Substituting from (4) into both sides of the preceding inequality, substituting the indicated copayments, and rearranging yields

$$
\frac{q_{1}-q_{0}}{q_{0}} \geq \frac{\left(k_{0}-k_{1}\right) h_{o}}{h_{a}-\left(p+s+a-k_{1}\right) h_{o}},
$$

where $q_{i} \equiv q\left(k_{i}\right)$. Substituting the calibrated parameters and copayments into (7) yields

$$
\Delta q \geq \frac{\left(0.20-k_{1}\right) h_{o}}{0.063-\left(5.25-k_{1}\right) h_{o}} .
$$

where $\Delta q \equiv\left(q_{1}-q_{0}\right) / q_{0}$. Intuitively, condition (8) says that the designer prefers the copayment if the market expansion on the left-hand side of the inequality exceeds the ratio of the health benefits bought with proceeds from the higher copayment (in the numerator on the right-hand side) to the incremental health benefit of a dose of PCV under the AMC compared to the same money being spent on an alternative intervention (in the denominator). The fact that the right-hand side of (7) is decreasing in $h_{o}$ implies that an increase in the opportunity cost of funds relaxes the condition, increasing the appeal of a lower copayment. In other words, a reduction in the opportunity cost of funds tightens the condition under which the designer prefers the lower copayment; lowering the copayment to $k_{1}$ would only makes sense if this boosts country take up sufficiently. Denote the threshold market expansion at which condition (8) just holds with equality by $\widehat{\Delta q}$.

Table 3 shows the market expansion required to make various lower copayments $\left(k_{1}=\right.$ $\left.\$ 0.10, k_{1}=\$ 0.05, k_{1}=\$ 0.00\right)$ preferable to $k_{0}=\$ 0.20$ given different level of the opportunity cost $h_{o}$. Across all table entries, the required market expansion is quite small to make lower copayments beneficial. 
TABLE 3-THRESHOLD MARKET EXPANSION FOR DESIGNER TO PREFER LOWER COPAYMENTS

\begin{tabular}{lcccc} 
& & \multicolumn{3}{c}{ Threshold quantity increase $\overline{\Delta q}$ for alternative copayments } \\
\cline { 3 - 5 } $\begin{array}{l}\text { Cost effectiveness of } \\
\text { alternative intervention, }\end{array} 1 / h_{o}$ (\$ per DALY) & $h_{0}$ & $k_{1}=\$ 0.10$ & $k_{1}=\$ 0.05$ & $k_{1}=\$ 0.00$ \\
\hline 4,914 & $2.035 \mathrm{e}-4$ & $0.03 \%$ & $0.05 \%$ & $0.07 \%$ \\
1,638 & $6.105 \mathrm{e}-4$ & $0.10 \%$ & $0.15 \%$ & $0.20 \%$ \\
1,000 & $1.000 \mathrm{e}-3$ & $0.17 \%$ & $0.26 \%$ & $0.34 \%$ \\
500 & $2.000 \mathrm{e}-3$ & $0.38 \%$ & $0.57 \%$ & $0.76 \%$ \\
150 & $6.667 \mathrm{e}-3$ & $2.33 \%$ & $3.53 \%$ & $4.76 \%$ \\
\hline
\end{tabular}

Notes: See previous table for rationales for cost-effectiveness measures. The second column is the reciprocal of the first. The last three columns substitute the respective values of $h_{o}$ and $k_{1}$ into third column into expression (8).

\section{B5.3. Speeding Rollout in Populous Countries}

According to Table 3, reducing co-payments to zero would be justified if this increased the number of children vaccinated by a mere $4.76 \%$ even using the cost effectiveness in the last row for the alternative intervention (\$150/DALY). To provide further context for this result, we will analyze conditions under which lowering the copayment would have been justified if it encouraged take up by populous countries like India and Bangladesh, which were late adopters under the AMC program.

Start with India, which did not participate in the AMC program until 2017 and then only in five states. The potential for market expansion can be gauged from India's experience with the HiB vaccine, introduced with GAVI support in 2013 in India. By our calculations, 79 million Indian children were immunized against $\mathrm{HiB}$ by 2018. Assuming that the coverage of PCV in India were similar, adding India to the program would have expanded the AMC market by $53 \%$ over the 143 million immunizations administered under the program by 2018.

This 53\% market expansion surpasses the $4.76 \%$ threshold by such a large margin that the copayment reduction would be justified even the expansion into the India market were uncertain, with a probability as low as $9 \%$ (where $9 \%=4.76 \% \div 53 \%$ ). This is true even though the expansion is limited to one country but extra cost per dose to make up for the reduced copayment is paid in all countries and across all periods in the model.

India rolled out PCV in five states in 2017. Calculations similar to those above can be used to show that if reducing the copayment to zero would have induced India to roll out PCV three 
years earlier in those five states, this alone would justify the extra cost (indeed, even if this market expansion were only $88 \%$ certain).

Turn to Bangladesh, which did not introduce PCV until 2015. If a copayment of zero would have induced Bangladesh to introduce the vaccine three years earlier, in 2012, this alone would justify the extra cost (indeed, even if this market expansion were only $82 \%$ certain).

\section{B5.4. Copayments by Vintage}

Having a lower copayment for new, more expensive vaccines than for older, cheaper vaccines may feel counterintuitive. Yet, countries may arguably have a greater elasticity of demand for newer vaccines than older ones because policymaking is subject to status quo bias and inertia. It may thus make sense to introduce new vaccines with a "free sample" policy.

\section{B5.6. Copayments as Market Test}

As well as contributing financing, copayments have another function, providing a "market test" for the AMC. This mitigates the problem of incomplete contracting when specifying the target of the AMC well in advance of production. If countries do not value the product when it is developed, no funds need to be expended under the AMC program.

Reducing the co-payment to zero removes this market test. A small copayment might therefore be beneficial, even if it is not justified by the calculations along the lines of Table 3 .

The market test is more important for technologically distant products, because it is both more difficult to specify their characteristics, and because the problem they intend to solve might not be there in future. For instance, an AMC for a malaria vaccine might stimulate the production of that vaccine. However, if malaria-carrying mosquitos are rendered extinct using genetic modification, then even a very good malaria vaccine will no longer be useful.

The PCV covered by the pilot AMC was a technologically close product, so the "market test” function of country copayments was less important there. Calculations along the lines of Table 3 are thus relevant for the pilot AMC, implying that lower copayments may have improved the efficiency of the program. 